\title{
Interactive comment on "Impacts of rainfall features and antecedent soil moisture on occurrence of preferential flow: A study at hillslopes using high-frequency monitoring" by Z. Peng et al.
}

\section{Anonymous Referee \#1}

Received and published: 13 April 2016

\section{General Comments:}

I have completed my review on the manuscript "Impacts of rainfall features and antecedent soil moisture on occurrence of preferential flow: A study at hillslopes using high frequency monitoring" by Z. Peng, H. Hu, F. Tian, Q. Tie, and S. Zhao. The paper tries to answer the question: how do rainfall features and antecedent soil moisture affect the occurrence of preferential flow on different hillslopes? Generally, the paper uses a quite new technique to evaluate the occurrence of preferential flow. Although the overall results of the paper are interesting, the presentation of the paper (English,

Printer-friendly version

Discussion paper 
structure of written text) is currently still lacking.

Title: second part of the title "A study at hillslopes ..." makes the overall title long and does not provide that much additional information about the content of the paper. My suggestion is to stick to a shorter version of the title.

Abstract: Some sentences are very long and make it hard to get the main message/ ideas of the paper. The paper would improve a lot if the text in the abstract is improved (some specific comments and technical comments regarding this section are given below). Additionally, the abstract should strive to more clearly summarize what the impact of the rainfall features + antecedent moisture conditions are on preferential flow - which factors affect the occurrence and how they affect the frequency/ occurrence of preferential flow.

Introduction: To create a stronger paper that more clearly demonstrates its "innovation" in the field, I would strongly suggest the authors to more rigidly convey the current limitations of previous research and the added role this paper plays to the discussion. Additionally it would help to clearly state what hypothesis you have - what you expected as an outcome of your study - and how your findings aid the field. At the moment it is not clearly stated what new concepts/ideas etc. are used (although the method used is e.g. not yet a standard method).

Introduction (2): Related to a comparison of results found in the literature: it makes it easier for the reader to generally know the methods used to analyze preferential flow in all examples. Sometimes the authors do this (e.g. mentioned that a column experiment was used), but critical information about the measurement setup fails (sensors used, dye tracers used, other tracers used?). Allaire et al (2009) - Quantifying preferential flow in soils: A review of different techniques - wrote a whole review on all techniques that could be used compare the results found, which can be used as a reference.

English language: currently, there are still a lot of grammar errors and strangely formulated sentences in the manuscript that make the manuscript less easy to read. Authors

Printer-friendly version

Discussion paper 
are advised to ask help from one or more native speakers to improve the level of English of the overall manuscript. - Authors differ between a rate based and a sequence based classification of preferential flow. I think it is important to realize that the extra use of a wetting velocity provides only an additional method to detect preferential flow. The method itself is not suitable to identify differences in preferential flow processes.

The separation between the results and the discussion is not clear. It seems like the results section still partly continues in the discussion part of the paper. Additionally, data is partly already discussed in the results section. When writing a separate discussion, this should only focus on the discussion of the results, not on the disruption of the results. A solution would be to (1) write a combined results and discussion section or (2) to better separate both sections and the aims of both separate sections of the paper.

Specific Comments:

Introduction:

Page 1, line 14: Please specify which frequency. I assume you relate to the frequency of preferential flow occurrence. Be more specific, otherwise this is unclear to the reader.

Page 1, line 15-16: Again, please specify that you refer to preferential flow frequency.

Page 1, line 16-17: "Antecedent soil moisture was also significantly correlated with the frequency. However, this should largely be attributed to the differences of soil moisture among sites, since varying range of soil moisture at a specific site was not wide enough to influence the frequency significantly". This is very unclear as the authors talk both about spatial (site-to site) and temporal (site specific range) soil moisture variability. I do not see how the spatial and temporal occurrence link. . .

Printer-friendly version

Page 1, line $26-28$ : I do not see how preferential flow can be densely distributed in soils. It is rather a process that is occurring, which either occurs or does not at a certain moment in time. 
Page 2, line 4: The authors write: "Among the many, rainfall features and antecedent soil moisture are two essential control factors ..." I noticed these ideas are used throughout the papers, forming the general framework of this paper. I think this is nice and agree with this. Nonetheless, I think it is important to define and accurately separate spatial and temporal components. Preferential flow can occur at a specific location, related to local soil moisture (and even rainfall- in case of vegetation - interception) conditions. As an example we might look at specific locations where preferential flow occurred more frequently and relate this to local conditions. At the same time, we can also look in time and specify temporal differences in precipitation and antecedent moisture, which might be related to seasonal/ climatic conditions at a specific point in time. In this case, we might look at the amount of sensor locations that responded under certain rainfall conditions.

Page 2, line 6: "Heppel divided ... intensity driven and duration driven". I think it only makes sense to make such a statement when explaining afterwards how and why he did this. Otherwise referring to this paper does not really convey a clear message and rather raises questions.

Page 2, line 13: "Wu et al. indicated ... growth rates along with increasing rainfall intensity". I think it is important to state here how this was measured, as one problem related to the use of soil moisture sensors is that changes in water content are not observed while the soil is saturated (see Graham and Lin, 2011 - Controls and frequency of preferential flow occurrence: A 175-event analysis; Wiekenkamp et al. 2016 - Spatial and Temporal Occurrence of Preferential Flow in a Forested Headwater Catchment; I even believe it is also mentioned in the Hardie (2013) paper). Nonetheless, preferential flow can still occur in reality. This is something to generally note/ keep in mind as a limitation of the method.

Printer-friendly version

Discussion paper
Page 3, line 14; Authors obtained information about summer canopy coverage using

\section{Methods:}


SPOT, August 2013). This is not sufficient to reconstruct how this information is obtained. Which satellite was used? SPOT 6? Additionally, it would be worthwhile to understand where the $98 \%$ comes from - is this the average over all pixels with in the catchment (also: specify resolution).

Page 3, line 18: "...from high to low". Please be more precise. Does this specify the height? If so, please specify that you are talking about altitude (one might confuse it with slope angles). Additionally, I wonder how the effect of canopy can be separated from the geomorphological location on the hillslope, as it seems that all non-vegetated monitoring locations are located on a relatively flat surface.

Page 3, line 21: the authors state that sensors were installed with different maximum depths .e.g. a different number of sensors per location. It would be worthwhile to know why? Was this related to the absolute depth of the soil/ the stone content in a given depth? Additionally, it would be important to know if the number of sensors influences the frequency of preferential flow as detected by the sensor response sequence.

Page 4, line 3: Authors state that soils are not that think, but afterwards mention that the soils are 0-2 meters deep. I could imagine that a 2 meter deep soil is not that shallow. To better understand if soils are generally shallow/deep, it would be important to state how deep soils are on average or what the characteristic thickness of soils is (could even be specified for different landscape positions).

Is there any information about the soil types that were found at the logger locations (using FAO or American Soil classification)? If available, it would be important to state such information here (and in the related Tables)

"Eighty four groups of soil samples..." Is this the number of soil samples or is this the number of groups - how many samples per group are there? I guess individual samples were meant here.

Page 4, line 8: Authors mention the usage of Rosetta to estimate Ks values. It would be

Printer-friendly version

Discussion paper 
important to state the function(s) used in the hierarchical artificial neural network model of ROSETTA - how are the measured soil physical parameters used to calculate Ks?

Ranges in soil properties per site are referred to in the methods section and given in table 2. What do the authors think about the factors that are the most influential for preferential flow occurrence? Is the rather range of hydraulic properties, the hydraulic properties of the most upper layer, or differences in hydraulic properties within the soil profile important for preferential flow occurrence?

Page 4, line 15-22: The determination of a rainfall event is commonly only defined by precipitation characteristics itself. In this case, the change in soil moisture at all depths is used. Why?

Page 4, line $15-22$ (2): The determination of a rainfall event relies on hourly thresholds. Is the original 10 minute resolution soil moisture and precipitation used for this approach or is the data aggregated to hourly values to determine the event start and end?

Page 4, line 24: Authors refer to the "Hardie et al. (2013) method". It is however unclear what type of method (the classification, mentioned later in the section or the wetting front velocities?)- What specific part of the analysis is referred to? Plus, it would be important to specify this here for reader that has not read the Hardie paper.

Page 5, line 2: Please replace "penetration velocity of the wetting front" by "wetting front velocity". Do this consequently - also for other parts in the manuscript. Additionally, one could question whether Eq. 1 needs to be written out here.

Results:

- Page 5, line 24: "Differences ... 46.8 mm". In which time frame? An hour/event/10 Printer-friendly version minute measurement/cumulative? - Page 6, line 3: "In order to compare ... selected". I wonder how the similarity of the events was examined. Should start and end date any of the events be the same for all rain gage locations? If not, how were "rainfall events 
observed by all rain gages" selected? - Figure 2: please specify the formula used for the curve that was fitted. And what was the RMSE of this fitted curve? In the text, the authors mention that they used a Pearson III curve. Please specify what type of curve is meant (I do not consider this a standard method). - Page 6, line 12: Differences between rainfall features were tested against the Gaussian distribution. Why? - Figure 4: I would prefer to see the data values in a table as it is difficult to infer the exact significance between sites. A table will additionally provide extra information (exact values). - 'Considering the rainfall events: Overall, it is not well specified which rainfall characteristics are used for a specific event. Where the average characteristics for all location used or are the location specific rainfall characteristics considered? Additionally: which rainfall data was used for the monitoring sites where no rainfall was measured? - Regarding figure 6: During both monitoring periods, the $\mathrm{FH}$ locations had several situations in which they all reacted preferentially. However, there is no situation in which all 12 sensor locations reacted at the same time. This would be an interesting point to bring up and discuss. Additionally, it is not that clear that the top four bars belong to period 1 and the lower 12 to period 2. To improve this, such information could be directly added in the figure. - On the statistics similarity of rainfall: only the similarity of the rainfall characteristics during the 39 simultaneous events was tested. Nonetheless, the events that were not occurring at the same time amongst all sites and that created local differences were not considered. Although these additional events/ variation is number of events do inform us about rainfall heterogeneities, they were "kicked out". Is it fair to afterwards state that precipitation differences did not influence the occurrence of preferential flow, although they might generate local differences amongst locations e.g. antecedent soil moisture conditions, canopy wetness.

\section{Discussion:}

Figure 7: It is not clear if the rainfall features vs. type of flow included all the specific rainfall amount for all individual events * the individual sites. If this is the case, it is logical that there is an overlap in characteristics, as Figure 6 already shows that individual

Printer-friendly version

Discussion paper
Interactive comment 
locations during the same event might cause different responses, which explains why similar rainfall conditions end up in the different classes.

Page 8, line 5:" The values ranges ...calculated". Frequencies here probably refer to the total number of sites that responded preferentially. It is important to mention such information specifically - e.g. if you integrated the data over time (to look spatially) or in space (to look temporally). Examples of papers that apply such approaches can be found in Liu and Lin (2015 - Frequency and control of subsurface preferential flow occurrence in the Shale Hills catchment: from Pedon to catchment scales).

Page 8, line 22: “..., $n=233$ ”. Where does this $n$ value come from? Where location specific rainfall conditions connected? Again, it is not clear how rainfall conditions were used - site specific or only event specific? The way this data is used should be better described throughout the manuscript.

Page 8, section "On the influence of antecedent soil moisture". Again, here it is important to state when antecedent soil moisture conditions were spatially or temporally used...

Figure 11, page 20: Why is the antecedent moisture not directly plotted against the frequency of preferential flow? This would better show the relationship between both variables.

Page 16, line 5 and page 20, line5: In these different figures (5 and 12), you visualize the soil moisture response to rainfall at different depths. Be consequent and use the same color scheme for both images. Generally, I think it is more difficult to follow the legend in the black and white images (what is what). Therefore, I would suggest either using different grey-tones or sticking to the colored figures.

\section{Technical Corrections:}

Introduction: technical comments were detailed described for the introduction (as a start and an example). Authors should however ask aid from a native speaker to check 
the manuscript more detailed.

Page 1, line 9: rephrase "observation was conducted ...." Additionally, you might need to specify what observations; this is not clear in this sentence, and it is part of the abstract, sentences need to be very clear (this is the part that is most read).

Page 1, line 12 "5-10 times of the saturated ..." Remove "of".

Page 1, line 9(end) rephrase "Totally ..."

Page 1, line 13: change "in average" to "on average".

Page 1, line 19 - 22: "Further examination suggested that topography and surface cover ... preferential flow". This sentence is too long and there are unclear connections - why does the sentence end with soil moisture where it started with the factors surface cover and topography?

Page 2, line 18: "though Hardie et al. (2013) suggested ..." This sentence build-up creates the idea that although Hardie et al. (2013) suggested it, other researchers do not agree. However, the agreement between authors/researchers is not in any way related to what Hardie et al. (2013) suggested. Please rewrite.

Methods/Results:

The authors use the word "rainfalls" multiple times in the manuscript (page 15, caption Figure 2, 3.1 results). There is no such thing as "rainfalls", as the plural form or "rainfall" does not exist. Please rephrase this throughout the manuscript.

Interactive comment on Hydrol. Earth Syst. Sci. Discuss., doi:10.5194/hess-2016-112, 2016. 